\title{
High-rate operation of the Micro-Hole and Strip Plate gas detector
}

\author{
J.F.C.A. Veloso ${ }^{a}{ }^{*}$, C.C. Caldas $^{\mathrm{a}}$, C.A.B. Oliveira ${ }^{\mathrm{a}}$, C.D.R. Azevedo ${ }^{\mathrm{a}}$, J.M.F. dos Santos ${ }^{\mathrm{b}}$, \\ A. Breskin ${ }^{\text {, }}$ R. Chechik ${ }^{\mathrm{c}}$ \\ ${ }^{a}$ Physics Department, University of Aveiro, 3810-193 Aveiro, Portugal \\ ${ }^{\mathrm{b}}$ Physics Department, University of Coimbra, 3004-516 Coimbra, Portugal \\ ${ }^{\mathrm{c}}$ Department of Particle Physics, The Weizmann Institute of Science, 76100 Rehovot, Israel
}

Available online 21 May 2007

\begin{abstract}
We report the performance of the Micro-Hole-and-Strip-Plate (MHSP) operated in xenon, under high-flux irradiation of a 30-kV $\mathrm{X}$-ray Cu-tube. Stable operation in pulse-counting mode for gas gains above $10^{4}$ was measured up to counting rates of $5 \times 10^{5} \mathrm{~Hz} / \mathrm{mm}^{2}$. A gain variation of less than $5 \%$ was observed for gains of $10^{4}$, while the energy resolution for 8 -keV X-rays degrades from $\sim 16 \%$ to $\sim 18 \%$. No visible degradation was observed on the MHSP irradiated area.

(C) 2007 Elsevier B.V. All rights reserved.
\end{abstract}

PACS: 29.40.Cs; 29.40.-n; 85.60.Gz

Keywords: MHSP; Count rate; Xenon

\section{Introduction}

Micropattern electron multipliers have been developed and studied for almost two decades, with applications to many different areas of knowledge, such as particle tracking, X-ray and neutron detection and imaging or diffraction experiments $[1,2]$. For most of the applications there is a continuous interest in developing high-count-rate detectors to cope with the high luminosity of particle and synchrotron radiation beams.

The small spacing between anode and cathode electrodes allows for small drift distances for positive ion collection, leading to fast transit times and decreased space charge effects, thus increasing the count-rate capabilities of the new gaseous detectors. In addition, the small pitch of patterned electron multipliers provides high spatial resolution, in the sub-mm range.

However, the maximum achievable gain drops with the count rate for all micropatterned detectors [3-5]. Moreover, for a given gain its value drops with increasing rate, at high count rates. Indeed, a fraction of the charge

\footnotetext{
${ }^{*}$ Corresponding author. Tel.: + 351239410667 ; fax: + 351234424965 .

E-mail address: jveloso@fis.ua.pt (J.F.C.A. Veloso).
}

produced in the avalanches induces charge build-up on the surface of the insulators that support the different electrodes, with consequent rate-dependent gain [e.g. Refs. 6-8].

A new electron multiplier, the Micro-Hole and Strip Plate (MHSP), has been recently proposed [9,10]. It integrates, on a single element, two successive independent charge amplification stages: a GEM-like and a MSGC-like stage. Like the GEM [11], the MHSP is fabricated with printed circuit board technology from a $50-\mu \mathrm{m}$ Kapton foil, metalized with 5- $\mu$ m-thick copper-layers on both sides. A GEM-like pattern of holes is etched through the foil with a continuous metal electrode on the top-side, and a standard microstrip pattern etched on the bottom side, with the holes centered on the wide cathode strips, Fig. 1.

The MHSP presents gains that are more than one order of magnitude higher than the single-GEM, reaching values well above $10^{4}$ in pure xenon [12], and fast signal response [10], $\sim 10-30 \mathrm{~ns}$. In addition, it has highly reduced ionbackflow, e.g. in a single MHSP more than $80 \%$ of the avalanche ions are trapped on the nearby cathodes [13]; this helps controlling ion-induced feedback effects.

Up to now, we had not yet investigated the performance of the MHSP operating at high count rates. While GEMs 

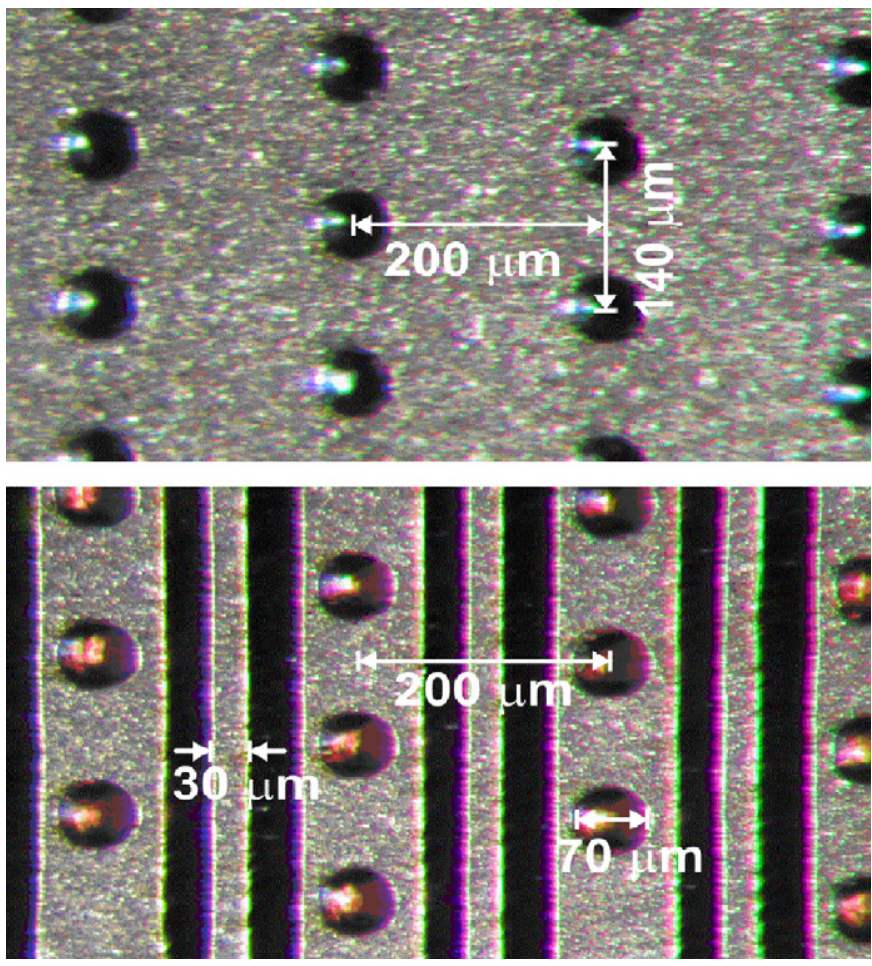

Fig. 1. Photomicrograph of the MHSP electron multiplier, top and bottom sides.

do not exhibit gain variations up to $10^{6} \mathrm{~Hz} / \mathrm{mm}^{2}$ [8], for standard MSGC the surface charge build-up effects play a significant role and a degradation in gain is already noticeable for rates as low as $10^{4} \mathrm{~Hz} / \mathrm{mm}^{2}[6,7]$.

In this work, we investigate the MHSP capability to operate under high-flux irradiation of 8-keV X-rays. Gain and the energy resolution degradation are monitored for counting rates up to $5 \times 10^{5} \mathrm{~Hz} / \mathrm{mm}^{2}$.

\section{Experimental set-up}

The present MHSP has an active area of $2.8 \times 2.8 \mathrm{~cm}^{2}$. The bi-conical holes, around $\varnothing 40 / 70 \mu \mathrm{m}$ are implemented in the Kapton/copper film, arranged in an asymmetric hexagonal lattice of 140 - and $200-\mu \mathrm{m}$ pitch in the directions parallel and perpendicular to the strips, respectively (Fig. 1). The anode and cathode micro-strip widths are 20 and $100 \mu \mathrm{m}$, respectively, with a $40-\mu \mathrm{m}$ gap between them and a $200-\mu \mathrm{m}$ pitch. All the MHSP electrodes are independently polarized, with the drift electrode at ground potential. The electric field in the holes is established through the voltage difference applied between the cathode strips and the top-electrode, $V_{\mathrm{CT}}$, while on the bottom-side of the MHSP an MSGC-like electric field is established through a potential difference between the anode and the cathode strips, $V_{\mathrm{AC}}$. The bottom mesh electrode was grounded and an electric field of $0.2 \mathrm{kV} / \mathrm{cm}$ was set in the drift region.

The MHSP operation principle is depicted in Fig. 2. Electrons produced by radiation in the gas volume above

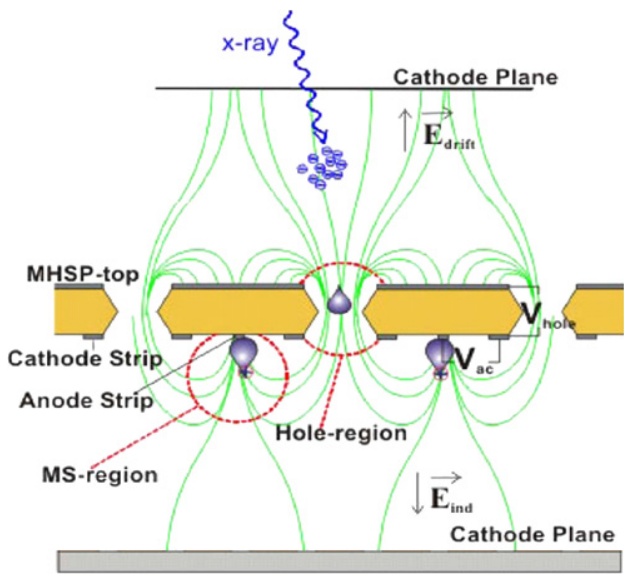

Fig. 2. Schematic diagram of the MHSP electron multiplier and its operation principle.

the MHSP (the drift region) are focused into the holes and undergo multiplication in the strong dipole electric field. The avalanche electrons are extracted towards the anode strip, in the MHSP bottom-side, where they are further multiplied in the strong electric field, and collected. The avalanche ions are mostly collected on the cathode plane and cathode strips. The drift and the induction regions are 5- and 3-mm thick, respectively.

The detector was irradiated with $\mathrm{X}$-rays from a copper $\mathrm{X}$-ray tube operated at $30 \mathrm{kV}$, placed at a distance of $30 \mathrm{~cm}$ from the detector. The X-ray beam was collimated to $\varnothing 0.5 \mathrm{~mm}$. The detector was filled with pure xenon at atmospheric pressure. Detector signals from the anode strips were fed through a Canberra 2006 charge preamplifier (sensitivity of $1.5 \mathrm{~V} / \mathrm{pC}$ ) and a Canberra linearamplifier $(0.5-\mu$ s shaping time) to a multichannel analyzer. The electronic chain sensitivity was calibrated for absolute gain measurement, using a calibrated capacitor directly connected to the preamplifier input and to a precision pulse generator.

\section{Experimental results and discussion}

In Fig. 3 we depict the detector relative gain and energy resolution as a function of the detector output count rate, for different MHSP absolute gains. The X-ray tube output flux was controlled varying the filament current. The values of $V_{\mathrm{CT}}$ and $V_{\mathrm{AC}}$ established for each gain are similar to those presented in Ref. [12]. No noticeable variation in the detector gain and energy resolution was observed for count rates up to $10^{5} \mathrm{~Hz} / \mathrm{mm}^{2}$. This demonstrates that MHSPs can withstand, without gain modifications, and at an avalanche size of $3.6 \times 10^{6}$ electrons (i.e. for gains of $10^{4}$ ), detected radiation rates of $10^{5} \mathrm{~Hz} / \mathrm{mm}^{2}$. For count rates of about $5 \times 10^{5} \mathrm{~Hz} / \mathrm{mm}^{2}$, i.e. the maximum rate that we could achieve with our X-ray tube, gain variations of only $5 \%$ were obtained for gains of $10^{4}$, and less than $2 \%$ for gains below $3 \times 10^{3}$. The energy resolution for high gains 
a

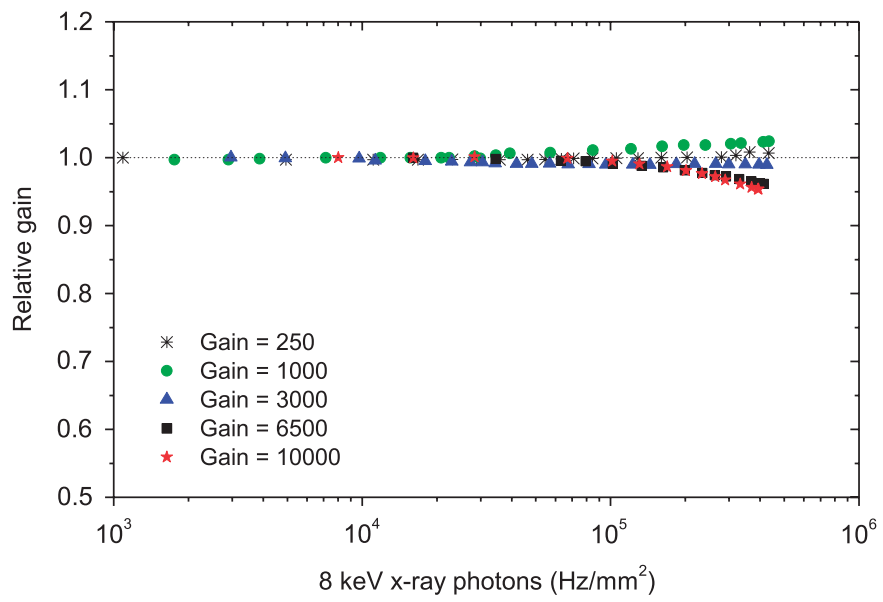

b

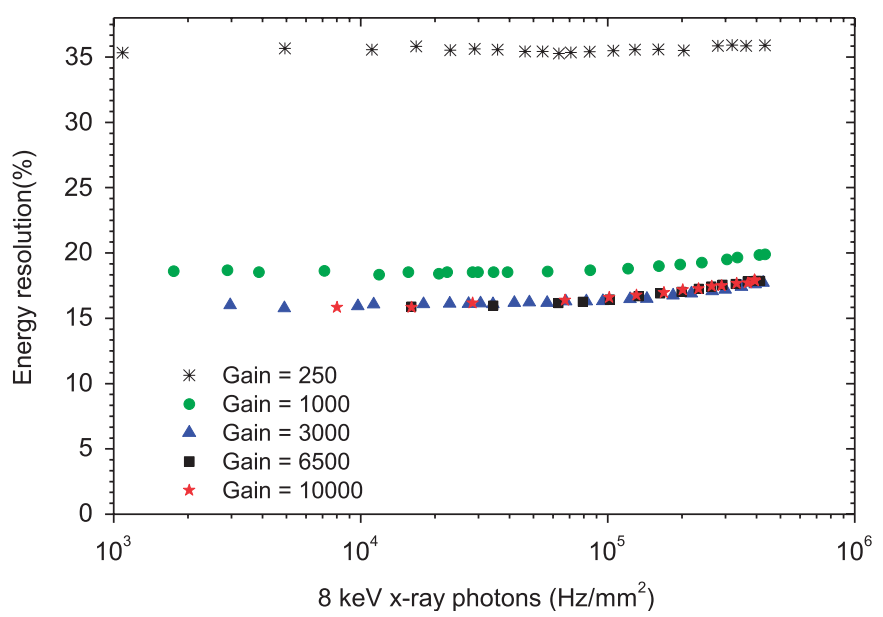

Fig. 3. Detector relative amplitude and energy resolution as a function of the detector output count rate, for different MHSP gains.

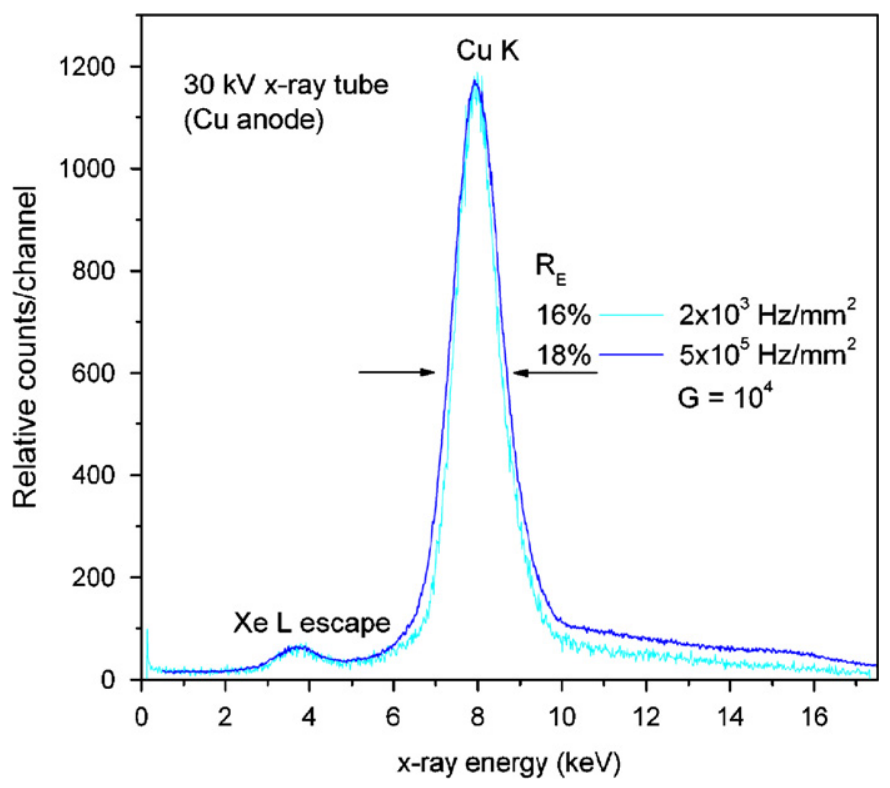

Fig. 4. Typical pulse-height distributions obtained for the X-rays emitted from the tube at low and high count rates and for a MHSP gain of $10^{4}$.

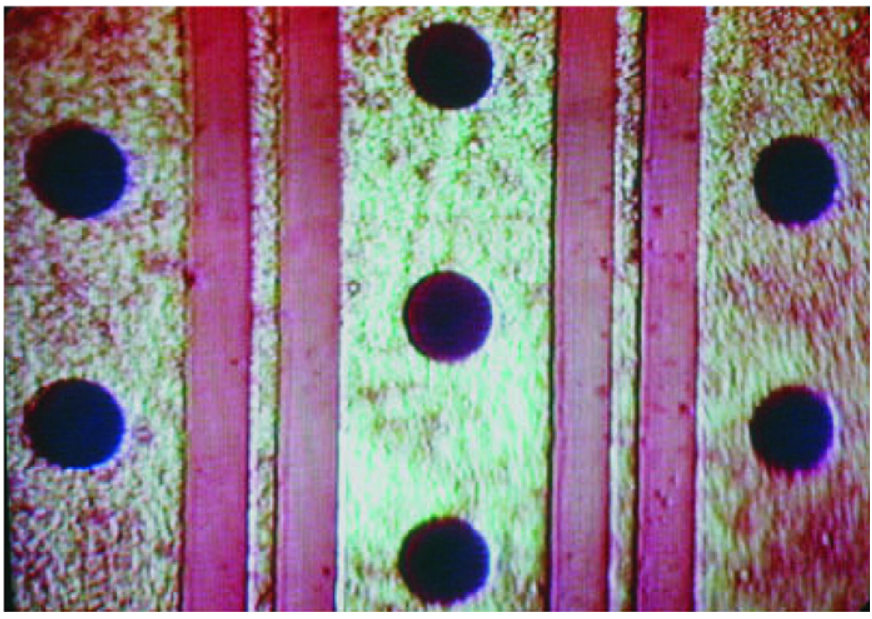

Fig. 5. Photomicrograph of a detail of the MHSP irradiated region.

degrades from $16 \%$ to $18 \%$ when the count rate increases from $10^{5}$ to $5 \times 10^{5} \mathrm{~Hz} / \mathrm{mm}^{2}$.

Fig. 4 presents two typical pulse-height distributions obtained for X-rays emitted from the tube at the maximum achievable count rate and, for comparison, at a low count rate $\left(2 \times 10^{3} \mathrm{~Hz} / \mathrm{mm}^{2}\right)$, for MHSP gains of $10^{4}$. A small amount of pile-up is observed in Fig. 4, contributing to the small degradation of the detector energy resolution.

The detector has been in continuous operation for several months, exposed to high rates for long periods, with no discernible radiation damage. The MHSP was exposed to charge rates in excess of $0.2 \mu \mathrm{C} \mathrm{mm}^{-2} \mathrm{~s}^{-1}$ for more than $1000 \mathrm{~s}$. After a detailed inspection, no damage was observed in the irradiated region of the MHSP, Fig. 5.

\section{Conclusions}

We have investigated the performance of the MHSP electron multiplier operated in pulse-counting mode under high rate irradiation of $8-\mathrm{keV} \mathrm{X}$-rays, in pure xenon at atmospheric pressure. The MHSP demonstrates stable operation for gains of $10^{4}$ and count rates of about $5 \times 10^{5} \mathrm{~Hz} / \mathrm{mm}^{2}$.

No visible variation of the detector gain and energy resolution was observed for count rates up to $10^{5} \mathrm{~Hz} / \mathrm{mm}^{2}$. For count rates of about $5 \times 10^{5} \mathrm{~Hz} / \mathrm{mm}^{2}$ it presented gain variations of only $5 \%$ for gains of $10^{4}$, and less than $2 \%$ for gains below $3 \times 10^{3}$. The energy resolution at high gains degrades from $16 \%$ to $18 \%$ when the count rate increases from $10^{5}$ to $5 \times 10^{5} \mathrm{~Hz} / \mathrm{mm}^{2}$.

No visible damage was observed in the irradiated area of the MHSP, after data taking during several months and exposure of the MHSP to charge rates in excess of $0.2 \mu \mathrm{C} \mathrm{mm}^{-2} \mathrm{~s}^{-1}$ for more than $1000 \mathrm{~s}$.

\section{Acknowledgments}

This work was supported by Project POCI/FP/63903/05, FEDER and FCT (Lisbon), and by the Israel Science 
Foundation project 151/01. A. Breskin is the W.P. Reuther Professor of Research in peaceful use of atomic energy.

\section{References}

[1] L. Shekhtmann, Nucl. Instr. and Meth. A 494 (2002) 128 and references therein.

[2] A. Sharma, 2000 IEEE Nuclear Science Symposium Conference Record, 2001, pp. 2-3.

[3] Yu. Ivaniouchenkov, et al., Nucl. Instr. and Meth. A 422 (1999) 300.

[4] Bressan, et al., Nucl. Instr. and Meth. A 424 (1999) 321.
[5] V. Peskov, et al., IEEE Trans. Nucl. Sci. NS-48 (2001) 1070 and references therein.

[6] J. Bohm, et al., Nucl. Instr. and Meth. A 360 (1995) 34

[7] F.D. van den Berg, et al., Nucl. Instr. and Meth. A 401 (1997) 156.

[8] J. Benlloch, et al., IEEE Trans. Nucl. Sci. NS-45 (1998) 234.

[9] J.F.C.A. Veloso, et al., Rev. Sci. Instr. 71 (2000) 2371.

[10] J.M. Maia, et al., Nucl. Instr. and Meth. A 504 (2003) 364.

[11] F. Sauli, Nucl. Instr. and Meth. A 386 (1997) 531.

[12] H. Natal da Luz, et al., Nucl. Instr. and Meth. A 552 (2005) 259.

[13] J.M. Maia, et al., Nucl. Instr. and Meth. A 523 (2004) 334. 